\title{
Multiplicative cascade models of multifractal solar magnetic fields
}

\author{
J. K. Lawrence, A. C. Cadavid, and A. A. Ruzmaikin \\ Department of Physics and Astronomy, California State University, Northridge, Northridge, California 91330
}

(Received 27 June 1994)

\begin{abstract}
We present a multifractal analysis of digital, photoelectric images of line-of-sight magnetic fields in solar active regions and quiet photosphere. We study a positive definite measure related to the Ohmic dissipation of magnetic energy. After calculation of the multifractal spectrum directly and by scaling of the moments of the measure, we focus on a multiplicative cascade approach. We infer a scale-invariant rule by which the Ohmic dissipation measure is allocated among subsets of its support through a hierarchy of scales. Knowledge of this rule, which is hampered to some extent by image noise, permits the calculation of the multifractal spectrum to great accuracy. The scaling of the solar dissipation field resembles that of fully developed turbulence in an atmospheric boundary layer. The cascade multiplier probability distribution is itself a very useful quantity. It allows a convenient display of image properties, such as self-similarity. Further, it is more closely related than the multifractal spectrum to the physics of the turbulent field evolution, and it thus can be used to impose stronger constraints on turbulent dynamo models of magnetic field generation.
\end{abstract}

PACS number(s): 47.53. $+\mathrm{n}, 96.60 . \mathrm{Hv}$

\section{INTRODUCTION}

Solar surface magnetic fields evolve in intimate connection with the turbulent motions of the photospheric plasma and ought, therefore, to share some of their properties. Fully developed turbulence possesses a scale invariance symmetry which is broken by spatial intermittence [1-6], and to some extent this property also characterizes magnetic fields in the solar photosphere [7,8]. A mathematical language suited to distributions with both scale invariance and intermittency is that of fractal geometry [9].

The solar magnetic field is distributed intermittently on its supporting set, so this scaling encompasses a spectrum of fractal dimensions [7,8]. Line-of-sight magnetic flux constitutes a signed measure. It has proven possible to demonstrate self-similarity in the corresponding solar flux distribution by means of a directly calculated multifractal spectrum, and this has led to a characterization of the solar field distribution as a "noisy multifractal" and has constrained acceptable dynamo models for small-scale field generation.

In this paper we consider a positive definite measure. The measure is associated with the Ohmic dissipation of magnetic energy and is analogous to the distribution of viscous dissipation often studied in connection with the scaling of fluid turbulence. Interestingly, this measure displays a high degree of scale invariance, while other choices, such as the field energy distribution, do not.

Multifractal measures are typically generated by means of a multiplicative cascade process. A measure is initially uniformly distributed over a set. It is then divided among subsets and then subdivided among sub-subsets, and so on to infinity, according to an invariant allocation rule $[10,11]$, which may be either deterministic or probabilistic. If the rule is known, and if the measure is scale invariant, then we can calculate the multifractal spec- trum exactly. This spectrum includes the effects of very large, and hence rare, intermittent fluctuations [12].

Here we hope to demonstrate the utility of the multiplicative cascade approach to multifractal analysis in the study of solar magnetism. Inevitably, when we deal with real data the degree to which the multiplicative cascade rule actually is known and actually is sale invariant is a matter of judgment. Image noise is an ever-present plague. As will be apparent below, a significant portion of our efforts has gone into attempts to estimate the degree and kind of noise and to make corrections for it.

Our data, in the form of digital, photoelectric images of line-of-sight solar magnetic fields, are described in Sec. II. In Sec. III we survey the multifractal formalism while reviewing some relevant aspects of past work. A measure related to the Ohmic dissipation of magnetic field energy is introduced. In Sec. IV the multiplicative cascade approach to multifractals is applied to the scaling of the Ohmic dissipation measure in images of both solar active regions and quiet Sun network magnetic fields. Some characteristics of the observed cascade multiplier distribution are interpreted in terms of image properties. In Sec. V we employ the multiplicative cascade approach to compare two fast dynamo models for photospheric field generation to observations, and results are discussed and conclusions offered in Sec. VI.

\section{OBSERVATIONAL DATA}

The primary data are two-dimensional, digital, photometric images of line-of-sight magnetic field. The data are obtained with the San Fernando Observatory $28 \mathrm{~cm}$, $f / 20$ vacuum telescope, and vacuum spectroheliograph system operated in the video spectra-spectroheliograph mode $[13,14]$. The images are derived from effective data "cubes" representing two spatial dimensions on the Sun 
and one spectral dimension. The observed spectral range includes the profile of the magnetically sensitive solar Fe I line at wavelength $630.25 \mathrm{~nm}$ (Landé $g=2.5$ ), a telluric $\mathrm{O}_{2}$ line at $630.20 \mathrm{~nm}$, and a partially blended telluric $\mathrm{O}_{2}$ line at $630.28 \mathrm{~nm}$. The spectral scale of the image cubes is $0.88 \mathrm{pm} \mathrm{pixel}^{-1}$. The east-west spatial scale is 0.46 arcsec pixel ${ }^{-1}$, corresponding to about $340 \mathrm{~km}$ on the Sun. The north-south spatial scale is similar, but depends on the spectroheliograph scanning rate. Actual spatial resolution is limited by atmospheric turbulence ("seeing") to $\geq 1$ arcsec. The field of view is $470 \times \sim 350$ pixels, corresponding to $\sim 200$ arcsec or $\sim 150000 \mathrm{~km}$ on the Sun.

The data cubes are reduced to spatial maps by operations along the spectral axis. The cubes are obtained in both left and right circular polarizations, which are con- verted to Stokes $I$ and $V$ line profiles allowing study of both the Zeeman and Doppler effects. The line-of-sight magnetic field in each pixel, equivalent to the line-of-sight flux averaged over the pixel area, is calculated from the first moment of the Stokes $V$ profile about the line center, divided by the equivalent width of the $I$ profile. We estimate "noise" in the images by the width of the weak-field Gaussian peak in a frequency histogram of the pixel field strengths. The $1 \sigma$ noise level ranges from $\sim 5$ to $\sim 15 \mathrm{G}$. Figure $1(\mathrm{a})$ is a line-of-sight magnetic field image including parts of two active regions (Nos. 6850 and 6853 in the National Oceanic and Atmospheric Administration's Solar Geophysical Data Bulletin) made under good seeing conditions with the video spectra-spectroheliograph system on 3 October 1991 at UT 20:03:30. Approximately
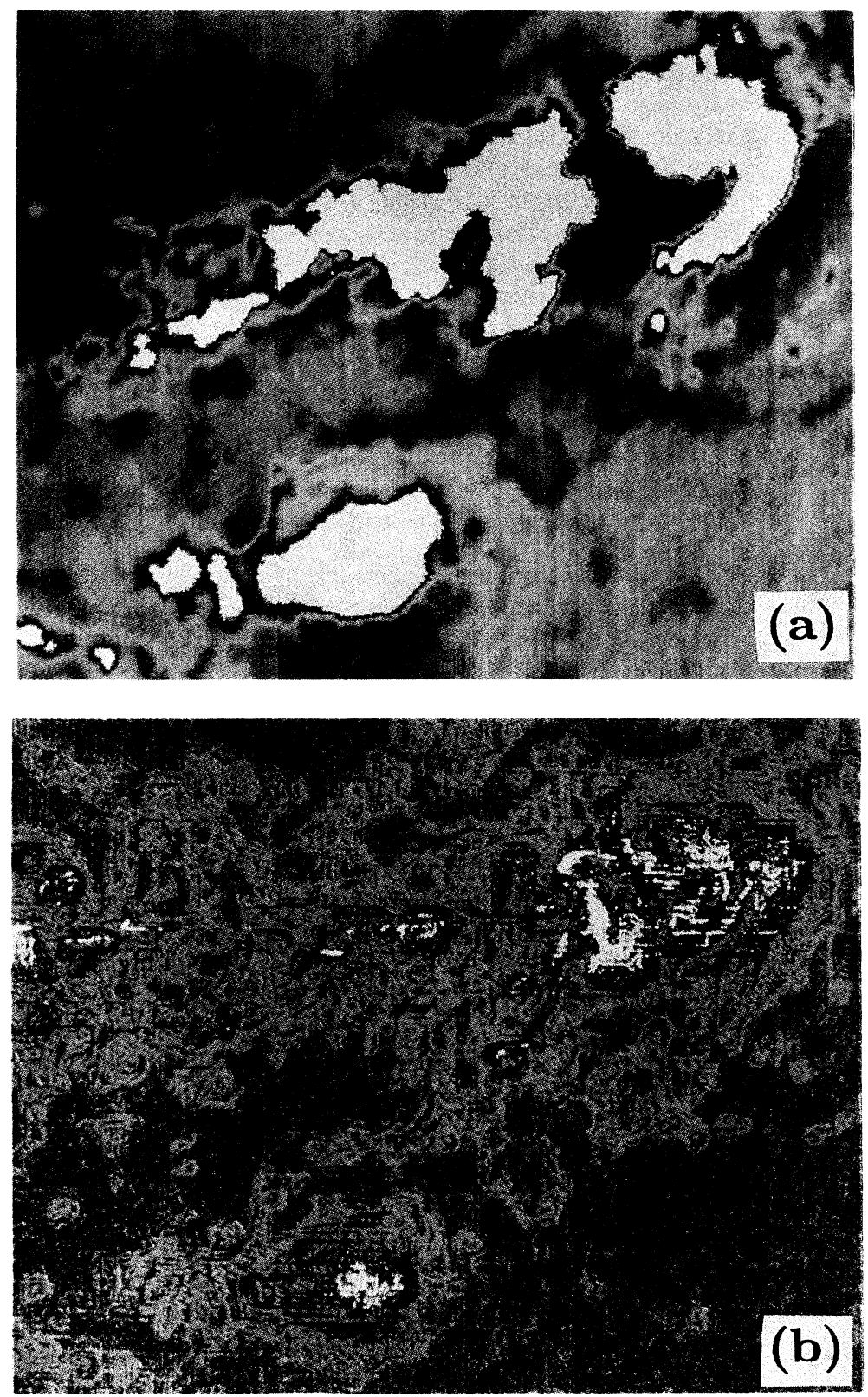

FIG. 1. (a) False color, line-of-sight magnetic field image of NOAA active regions 6850 and 6853 made with the San Fernando Observatory video spectra-spectroheliograph system on 3 October 1991 at UT 20:03:30. The spatial sale is 0.46 arcsec pixel $^{-1}$, corresponding to about $340 \mathrm{~km}$ on the sun. Spatial resolution is seeing limited at $\geq 1$ arcsec. The field of view is $\sim 200$ arcsec or $\sim 150000 \mathrm{~km}$ on the sun. White (black) represents positive (negative) magnetic polarity. North is up, east is left. (b) Ohmic dissipation measure derived from image (a) via Eq. (3.7). 
1000 such image sets have been accumulated up to the time of writing.

Other data examined included two very high-resolution images made by the $50-\mathrm{cm}$ Vacuum Solar Telescope and Lockheed tunable filter instrument at the Swedish Solar Observatory, La Palma [15]. These were made under the best seeing conditions and demonstrate 0.5 -arcsec resolution. The images are composed of $512 \times 512$ pixels of 0.14 -arcsec scale. Field of view is 85 arcsec or $\sim 60000$ $\mathrm{km}$ on the Sun.

Also studied were images from the 50-cm Swedish Vacuum Solar Telescope which were reconstructed by speckle interferometry so as to reach the instrument's diffraction limit of resolution at $0.22 \operatorname{arcsec}$ [16]. These images are made on a time scale shorter than that of much of the turbulent motion of the Earth's atmosphere. Then the effects of atmospheric spatial inhomogeneities are ameliorated by numerical methods. These images are composed of $130 \times 104$ pixels of 0.063 -arcsec scale. The field of view is thus about 8 arcsec or $\sim 5500 \mathrm{~km}$ on the Sun.

\section{SCALING AND SELF-SIMILARITY OF A MAGNETIC IMAGE}

\section{A. The box counting procedure}

We consider the set of pixels in a digital image of the solar surface which contain some measure. This might be line-of-sight magnetic flux, a signed measure [7,17]. An alternative, positive definite measure is the Ohmic dissipation of magnetic energy. The images, illustrated in Fig. 1, constitute a Euclidean embedding space for the sets, with dimension $d=2$ and linear size, say, $L_{1} \times L_{2}$. To examine image scaling, we cover the image with a uniform grid of boxes of side $s \equiv \epsilon \sqrt{L_{1} L_{2}}$ with $\epsilon \ll 1$. The "coarse-grained" measure $\mu_{i}(\epsilon)$ in the $i$ th box is calculated by adding the individual measures of each pixel in the box. If the measure is signed, the opposing components are allowed to cancel within each box and then the absolute value is taken. At each coarse-graining level (value of $\epsilon$ ) the measure is normalized to a unit total value over the image (this occurs naturally for a positive definite measure). In actual calculations we employ a Monte Carlo method in which the lattice is randomly sampled with boxes of a given size. This allows the accumulation of good counting statistics, even for relatively large boxes, and it averages over many possible partitions of the image.

We are interested in statistical properties of the measure, such as self-similarity, as the box size $s$, or $\epsilon$, is varied. For example, we can count the number $N(\epsilon)$ of boxes at the coarse-graining scale $\epsilon$ which contain any flux. If, on varying $\epsilon$, we find the power law $N(\epsilon) \propto \epsilon^{-D}$ over a finite scaling range, then the invariant quantity $D \leq d$ is the fractal dimension of the set.

\section{B. Direct calculation of the multifractal spectrum}

The fractal dimension refers to the geometric distribution of the elements of a set. The concept of "multifrac- tality" refers to a measure defined on the set; it takes into account the differing amounts of magnetic flux or of Ohmic dissipation contained in the pixels and hence in the coarse-graining boxes. The measure in the $i$ th box at scale $\epsilon$ is cast in power law form

$$
\mu_{i}(\epsilon)=\mu\left(\alpha_{i}, \epsilon\right)=\epsilon^{\alpha_{i}}
$$

Because $\epsilon<1$, large values of $\alpha_{i}$ correspond to small values of $\mu_{i}$. To characterize the distribution of the measure, for each coarse-graining scale $\epsilon$ we make a histogram of the number $d N(\alpha, \epsilon)=n(\alpha, \epsilon) d \alpha$ of boxes with "singularity strength" $\alpha$ in bins of width $d \alpha$. If the number density $n(\alpha, \epsilon)$ scales according to an approximate power law

$$
n(\alpha, \epsilon)=\phi(\ln \epsilon) \epsilon^{-f(\alpha)},
$$

where the "multifractal spectrum" $f(\alpha)$ is independent of $\epsilon$ in the limit $\epsilon \rightarrow 0$, then the measure is self-similar and multifractal according to the criterion of Evertsz and Mandelbrot [18]. When it has a positive value $f(\alpha)$ plays the role of fractal dimension of the subset of boxes with singularity strength $\alpha . f(\alpha)$ is normally concave downward; its maximum $f\left(\alpha_{0}\right)=D$ is the fractal dimension of the supporting set of the measure.

When applied to the line-of-sight fields, this approach displays the presence of image noise in an easily identifiable and even analytically treatable way [8]. This has permitted the demonstration of self-similarity in the solar field distributions. However, ambiguities [19] in the function $\phi(\ln \epsilon)$ render this method inappropriate for precise calculation of the multifractal spectrum.

\section{Scaling of moments of the measure}

The most common approach to multifractals is through the scaling of moments of the measure $[1,4]$. Starting with the measure $\mu$, we calculate the "partition function"

$$
Z(q, \epsilon) \equiv \sum_{i} \mu_{i}^{q}(\epsilon)=A(q) \epsilon^{\tau(q)},
$$

where the sum is over the boxes at the coarse-graining level $\epsilon$.

If for all $q$ in the limit $\epsilon \rightarrow 0$ the partition function scales with exponent $\tau(q)$ independent of $\epsilon$, then the measure is multifractal. For real data $s=\epsilon L$ cannot be smaller than a single pixel, so we cannot directly take the limit $\epsilon \rightarrow 0$. If, however, $\ln Z(q, \epsilon)$ depends linearly on $\ln \epsilon$ over some scaling range, then $\tau(q)$ may be taken to be the corresponding slope. This permits extrapolation below the resolution limit. For our magnetic images we find

$$
\frac{\ln Z(q, \epsilon)}{\ln \epsilon}=\left[\tau(q)+\frac{\ln A(q)}{\ln \epsilon}\right] .
$$

The partition function can be expressed as an integral over the singularity spectrum in Eq. (3.2):

$$
\begin{aligned}
Z(q, \epsilon)=\sum \mu^{q} & =\int n(\alpha, \epsilon) \epsilon^{q a} d a \\
& =\int d \alpha \phi(\epsilon) \epsilon^{q \alpha-f(a)} \propto \epsilon^{\tau(q)} .
\end{aligned}
$$


In the limit $\epsilon \rightarrow 0$ the entire contribution of the integral comes when $q \alpha-f(\alpha)=\tau$ is a minimum. Thus if, and only if, $f^{\prime \prime}(a)<0$, the quantities $\alpha, f(\alpha)$, and $q, \tau(q)$ are connected by a Legendre transformation

$$
\alpha=\frac{\partial \tau(q)}{\partial q}, f(\alpha)=q \alpha-\tau(q) .
$$

For a pure fractal these curves reduce to the single point $f(\alpha)=\alpha=D$. Large (small) values of $q$ correspond to small (large) values of $\alpha$.

Because of image noise this approach is essentially unusable for the signed measure of the solar data. The presence of a component of zero-centered Gaussian noise in such a measure can be shown to cause $\tau(q)$ to diverge for all $q \leq-1$. Further, the noise frequently changes the sign of $f^{\prime \prime}(\alpha)$ over a key range of $\alpha$, rendering the Legendre transformation invalid. This problem is ameliorated when we go instead to a positive definite measure.

\section{The magnetic dissipation measure}

Because it is central to the nature of Kolmogorov scaling, many studies of the multifractal nature of fluid turbulence focus on the distribution of viscous dissipation of the turbulent energy. Here we consider an analog for solar photospheric magnetism which is related to the Ohmic dissipation of field energy. This is proportional to $\mathbf{j} \cdot \mathbf{j}$, where the current density is calculated from the observed fields: $\mathbf{j} \propto \boldsymbol{\nabla} \times \mathbf{B}$. From two-dimensional, line-of-sight magnetic images made near disk center, we have knowledge only of $B_{z}(x, y)$ and will therefore use a partial measure defined at the single pixel level according to

$$
\begin{aligned}
\mu(i, j)= & {\left[B_{z}(i+1, j)-B_{z}(i-1, j)\right]^{2} } \\
& +\left[B_{z}(i, j+1)-B_{z}(i, j-1)\right]^{2} .
\end{aligned}
$$

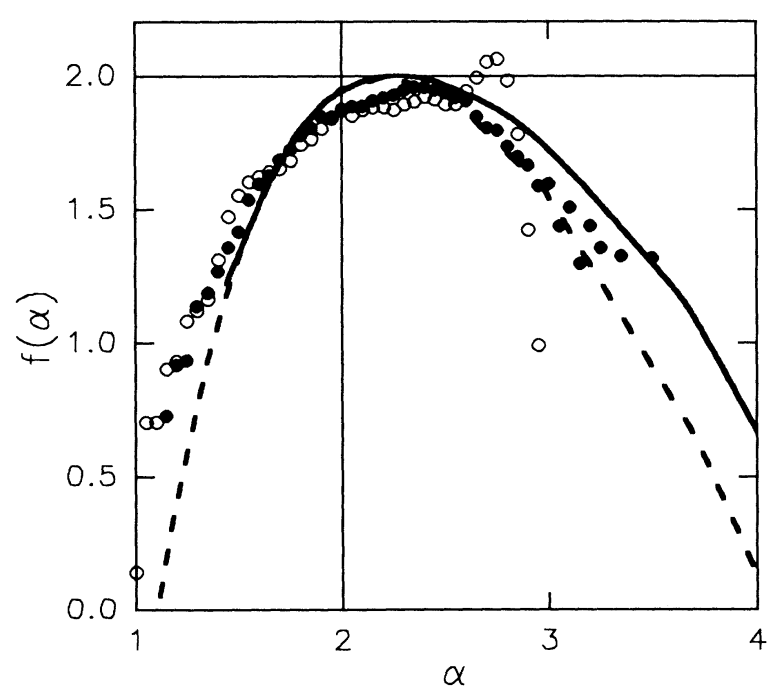

FIG. 2. Multifractal spectrum versus singularity strength for the measure of Fig. 1(b). The open circles denote curves constructed directly from Eq. (3.2) for $\epsilon=0.0025$, filled circles for $\epsilon=0.04$. The solid curve is calculated from moments of the measure by Legendre transformation and the dashed curve is calculated via the multiplicative cascade model.
Figure 1(b) is a map of this dissipation measure derived from the image of Fig. 1(a). In the lower and middle photosphere, observed fields are thought to be contained in thin, vertical flux tubes [20]. In this approximation, the above measure represents the dominant part of the dissipation.

Figure 2 shows the $f(\alpha)$ curves for the dissipation measure $\mu$ constructed directly from Eq. (3.2) for $\epsilon=0.0025$ and 0.04 . The left-hand sides of the curves, corresponding to the strong concentrations of dissipation, converge well to a single curve. The complex structure on the right-hand side of these plots represents the effect of image noise. The solid line in Fig. 2 is $f(\alpha)$ calculated via the Legendre transformation as described above. The dashed line is the singularity spectrum calculated by means of a "random multiplicative model" as described below.

\section{RANDOM MULTIPLICATIVE MODELS}

\section{A. Multiplicative processes and the multifractal spectrum}

A class of cascade models produces positive definite measures with multifractal scaling $[5,10,11,21]$. These begin with a measure distributed uniformly on a set. The measure is then allocated among given subsets according to either a deterministic or a probabilistic rule. For example, the measure on the unit interval might be divided between the two halves of the interval, either according to a fixed multiplier (say, $M_{0}$ and $1-M_{0}$ ) or according to a multiplier selected at random from a known probability distribution $P(\boldsymbol{M})$, where conservation of the measure requires that $P(M)=P(1-M)$. The deterministic case, of course, corresponds to a $\delta$-function probability density $P(M)=0.5\left[\delta\left(M-M_{0}\right)+\delta\left(M-1+M_{0}\right)\right]$. Next, the measure contained in the subsets is divided again among sub-subsets according to the same rule. Then the measure is again divided among sub-subsubsets, and so on to infinity. The invariance of the allocation rule through all the levels of subdivision produces multifractal scaling. We can compute $f(\alpha)$ from the moments $\left\langle M^{q}\right\rangle$ of $P(M)$, where $q$ is a continuous parameter $[6,10,11]$.

In an isotropic, two-dimensional version of this procedure, we begin with a square area uniformly filled with a unit measure. The measure is divided between, say, the left and right halves of the square according to the multiplier $M_{1}$ and $\left(1-M_{1}\right)$. Then the measure on the lefthand side is divided into two equal squares according to the multiplier $M_{2}$ and $\left(1-M_{2}\right)$ and the right-hand side is divided into two equal squares according to $M_{3}$ and $\left(1-M_{3}\right)$. Thus the unit measure in the original square is divided among the four half-size subsquares, which now contain the measures $M_{1} M_{2}, M_{1}\left(1-M_{2}\right),\left(1-M_{1}\right) M_{3}$, and $\left(1-M_{1}\right)\left(1-M_{3}\right)$, respectively. The multipliers $M_{1}$, $M_{2}$, and $M_{3}$ ar selected at random from the same symmetric distribution density $P(M)$. In the next step, each of the four squares is further divided into four subsquares according to the same procedure and with the same probability density. Then these subsquares are further subdivided, and so on. Because $P(M)$ is fixed at all levels of the subdivision, the limiting measure is multifractal. 
Also as a result of this scale invariance, the moments of the measure over the whole image can be reduced to the moments averaged over many realizations of a single subdivision:

$$
\left\langle M^{q}\right\rangle=4\left[\int_{0}^{1} d M P(M) M^{q}\right]^{2} .
$$

This correctly gives the normalizations $\left\langle M^{0}\right\rangle=1 / \epsilon^{2}=4$ and $\left\langle M^{1}\right\rangle=1$ reflecting the conservation of the unit measure. By analogy with Eq. (3.4) we obtain the scaling exponent of the partition function by setting $\epsilon=\frac{1}{2}$,

$$
\langle\tau(q)\rangle=-2-\frac{2 \ln \left\langle M^{q}\right\rangle}{\ln (2)} .
$$

Now the Legendre transform gives

$$
\langle\alpha(q)\rangle=\frac{d}{d q}\langle\tau(q)\rangle=-\frac{2\left\langle M^{q} \ln M\right\rangle}{\left\langle M^{q}\right\rangle \ln (2)}
$$

and

$$
f(\alpha)=q\langle\alpha(q)\rangle-\langle\tau(q)\rangle .
$$

We have verified for an assumed probability distribution $P(M)$ that the singularity spectrum calculated analytically by this procedure matches that found by numerical analysis from the corresponding generated image.

\section{B. Multiplicative process from the data}

The central element in the cascade process is the scale invariant probability distribution $P(M)$. We estimate this function from our two-dimensional data as follows. First we choose a scale of $s=2 n$ pixels. We then located a square box of this size at random in the image in question, divide this into four equal boxes of side $n$, and determine the ratio of measure in the upper two boxes to each other, then the lower two, the left two, and the right two. This gives four sample values of $M$. The choice of box is repeated many times to build up a distribution. Then a new scale is selected and a new distribution built up, and so on. Symmetry of $P(M)$ about $M=0.5$ is forced by the assumed conservative nature of the cascade process. Because of the details of our image generation process, described above, we also must check that the allocation of measure between the left and right halves of the original square, and between the top and bottom halves, also is given by the same $P(M)$. We find this to be so, within reasonable tolerance. We have also used a two-step process in which we first partition the original square box into equal rectangles and then partition the rectangles into equal squares. Each of these steps is equivalent to a decrease of $\epsilon$ by a factor $\sqrt{2}$ in Eq. (4.2). At each of these stages we obtain sample values of $M$ by taking the ratio of the measure in each partitioned area to that of its parent. The $P(M)$ encountered via this method is, within a small margin of error, the same as that from the previous method.

Figure 3(a) shows $P(M)$ for the dissipation measure of Fig. 1(b) for $s=4,6,8,12,16,24,32$, and 48 pixels (for $s=4, n=s / 2=2$ pixels corresponds roughly to the resolution scale). This displays a high degree of scale invariance. Shown in Fig. 3(b) is the equivalent $P(M)$ from a second active region image obtained at the San Fernando Observatory. These images have a pixel scale of 0.46 arcsec and resolution $>1$ arcsec. Figure $3(\mathrm{c})$ is derived from a Lockheed-La Palma magnetic image of active region plage with pixel scale of 0.14 arcsec and resolution $\approx 0.5$ arcsec [15]. Figure $3(\mathrm{~d})$ is derived from a speckle interferometric reconstruction of a La Palma image of active region plage. The pixel scale is 0.063 arcsec and the resolution is diffraction limited at $\approx 0.2 \operatorname{arcsec}[16]$. We
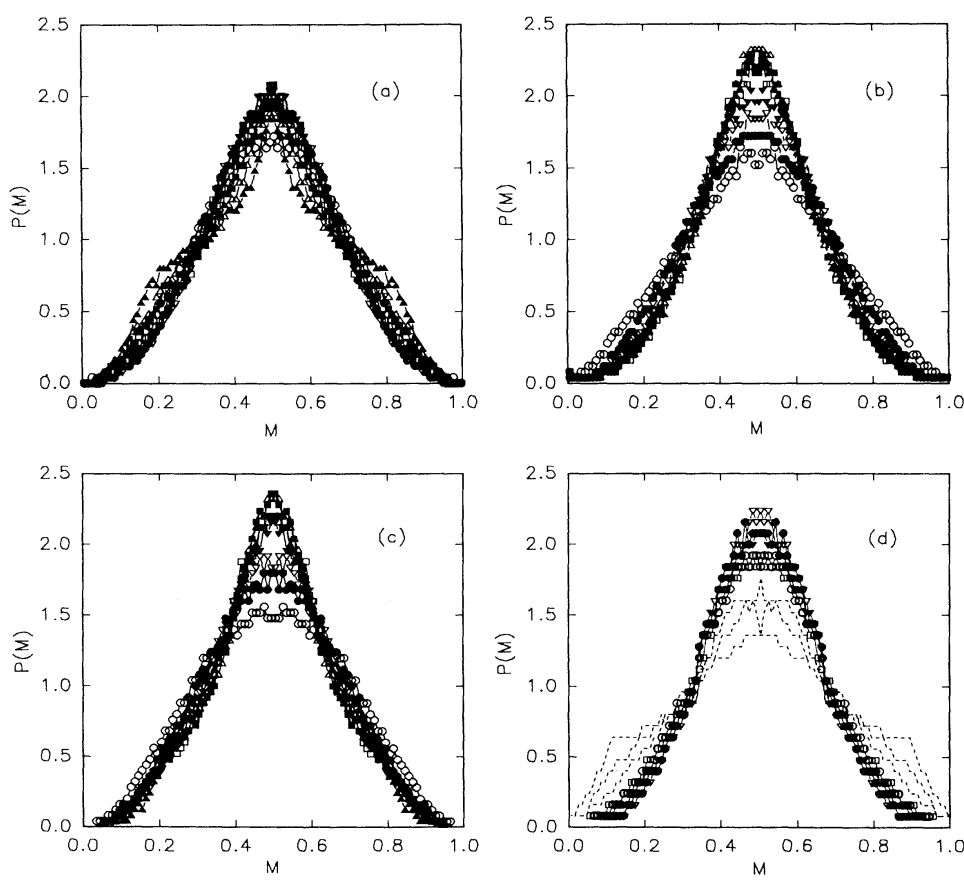

FIG. 3. Cascade multiplier probability distribution density versus multiplier for $s=4$ (open circles), 6 (filled circles), 8 (open down triangles), 12 (filled down triangles), 16 (open squares), 24 (filled squares), 32 (open up triangles), and 48 pixels (filled up triangles). (a) For the measure of Fig. 1(b) with a pixel scale of 0.46 arcsec and a resolution $\geq 1$ arcsec. (b) From a second San Fernando Observatory active region image. (c) From a Lockheed-La Palma magnetic image of active region plage made on 6 June 1990 with a pixel scale of 0.14 arcsec and a resolution $\approx 0.5$ arcsec. (d) From a speckle interferometric reconstruction of a La Palma image of active region plage. The pixel scale is 0.063 arcsec and the resolution is diffraction limited at $\approx 0.2$ arcsec. Here the curves for $s=24,32$, and 48 pixels are shown only as dashed lines. 

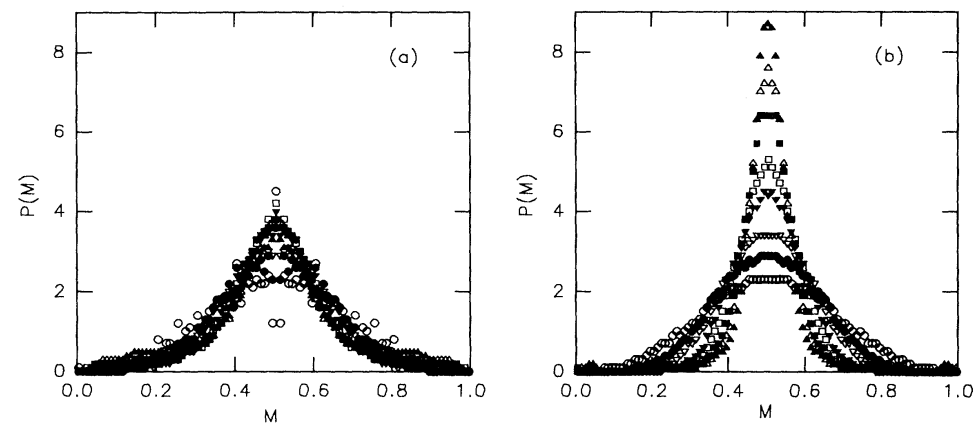

FIG. 4. Cascade multiplier probability distribution density versus multiplier for the dissipation measure in San Fernando Observatory images of quiet Sun network fields made on 9 September 1993 for $s=4$ (open circles), 6 (filled circles), 8 (open down triangles), 12 (filled down triangles), 16 (open squares), 24 (filled squares), 32 (open up triangles), and 48 pixels (filled up triangles). (a) An average over 24 images made at 10 -min time intervals. (b) A single image. note that this image is only $\approx 100$ pixels on a side, so that poor counting statistics can account for the loss of invariance at the largest scales.

Figure 4 illustrates the probability $P(M)$ for a San Fernando Observatory image of quiet Sun network fields. Figure 4(a) gives the result for an average over 24 images made at 10-min time intervals; Fig. 4(b) gives the result for a single image. Because the quiet Sun fields contain relatively little magnetic flux, they may be dominated by image noise. Furthermore, the fields themselves evolve slowly in the quiet Sun. Thus the effect of noise may be reduced by averaging over a sequence of images taken close together in time. A comparison of Figs. 4(a) and 4(b) indicates that the result of reducing the noise is to reduce the non-scale-invariant central peak of the $P(M)$ distribution and to broaden its base, thus producing rough scaling. This implies that the lesser, non-scaleinvariant central peaks in the active region images, especially in Figs. 3(b) and 3(c), may be the result of image noise. The larger the scale the more exaggerated the effect of the noise, which lowers the wings of the curves and produces a strong, narrow central peak. The curve for the smallest scale $s=4$ pixels is only very weakly affected by the presence or absence of noise and it is tempting to speculate that this may represent the true zero-noise limit, both in Figs. 3 and 4 . In the following calculations, however, we have averaged over scales. The image averaging technique is not appropriate for active region data. First, the image noise accounts for only a small fraction of the total measure. Second, significant evolution of the field structures is seen to occur within the time span of the image sequence. The result of averaging produces an image which, rather than scale invariant, is too uniform $[P(M)$ highly peaked $]$ at small scales and too intermittent $[P(M)$ very broad] at large scales.

\section{Multifractal spectra from the data}

Figure 5 shows the $f(\alpha)$ multifractal spectra calculated via Eqs. (4.1)-(4.4) from the scale averaged probability densities $P(M)$ of Figs. 3 and 4. These curves are quite similar, despite the differing physical scales of the four images. Note that they extend at both ends to $f(\alpha)=-\infty$. A detail of the multifractal spectrum for the case of Fig. 3(a) is shown as the dashed line in Fig. 2. This is in good agreement with the spectra computed via other methods on the left-hand, strong-field side of the plot. The generator differences on the right-hand, weakfield side can be attributed to the effect of image noise.

The form of the $P(M)$ distributions in Figs. 3 and 4 resembles that found by a similar procedure for onedimensional data on a turbulent dissipation field in an atmospheric surface layer [11]. The corresponding $f(\alpha)$ curves provide a more explicit indication of the possible universality of our result. Concentrating on a particular value $q=2$ in Eqs. (4.1)-(4.4), Chhabra and Sreenivasan [22] found significant sample-to-sample fluctuations in the values of $f(q=2)$ and $\alpha(q=2)$ for ten simulations using a binary cascade model with triangular $P(M)$ distribution and for ten samples of fully developed turbulence in an atmospheric boundary layer. Further, the twenty values of $f(2)$ showed a strongly linear dependence on the value of $\alpha(2)$. Such behavior is not characteristic of deterministic systems. We have calculated $f(2)$ and $\alpha(2)$ for the five solar images represented in Figs. 3 and 4. These also show linear sample-to-sample

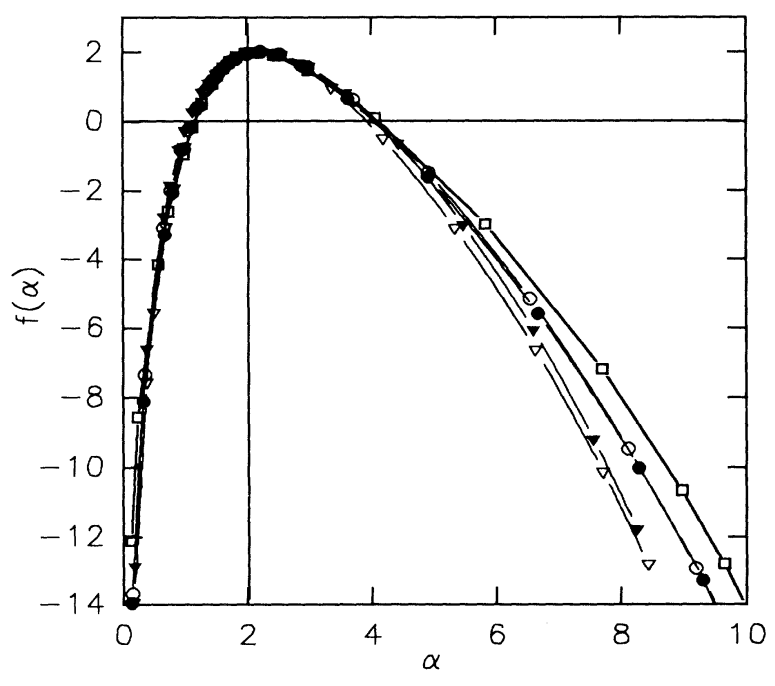

FIG. 5. Multifractal spectra versus singularity strength calculated from the scale averaged $P(M)$ of Figs. 3(a), San Fernando 3 October 1991 (open circles); 3(b), San Fernando 17 August 1989 (filled circles); 3(c), Lockheed 6 June 1990 (open triangles); 3(d), Speckle (filled triangles); and 4(a), San Fernando quiet Sun 9 September 1993 (open squares). 
fluctuations. In addition, when the results are converted to an effective one-dimensional form by letting $f \rightarrow f / 2$ and $\alpha \rightarrow \alpha / 2$, the five points lie in the same linear band found for the simulations and for atmospheric turbulence.

\section{Interpretation of probability distribution}

If the image being analyzed is completely uniform, then the measure will always be divided equally between adjacent squares and we will have $M=0.5$ in every case. Then $\boldsymbol{P}(\boldsymbol{M})=\delta\left(\boldsymbol{M}-\frac{1}{2}\right)$. Thus a narrow distribution, peaked about $M=0.5$, indicates a relatively uniform measure. On the other hand, an intermittent measure will have a broader $P(M)$. If the measure contains empty holes, then the distribution will be nonzero at $M=0,1$. From this it is apparent that the addition of noise to an image with holes will tend to fill the holes and thus decrease the values of $P(0)=P(1)$.

In the case of the $P(M)$ in Fig. 3(a) from our image of Fig. $1, P(M)$ goes to zero at some $M^{*}>0$. Thus the moments in Eq. (4.1) are finite for all $q$. This means that when we calculate the $f(\alpha)$ spectrum the whole range of values $q \in[-\infty,+\infty]$ will be represented. If, however, $P(M)>0$ for $M=0$, then the integrand in Eq. (4.1) will diverge for all $q$ less than or equal to some finite value $q_{0}$. This means that $\alpha(q) \rightarrow \infty$ and $f(\alpha) \rightarrow-\infty$ for $q=q_{0}$ and only moments $q \in\left[q_{0},+\infty\right]$ figure in the scaling. In the case of a uniform distribution $P(M)=1, M \in[0,1]$, we find $q_{0}=-1$. If $P(M)$ goes to zero when $M \rightarrow 0$, then the convergence of the integral in Eq. (4.1) depends on its functional form there, that is, on the order or strength of th holes in the measure. If $P(M) \propto M^{p}$, where $p \neq 0$, near $M=0$, then we find $q_{0}=-1-p$. If, for example, $p=1$, which approximates the case observed by Chhabra and Sreenivasan [11] for turbulence in an atmospheric boundary layer, then the spectrum $f(\alpha)$ encompasses moments only for $q \in[-2,+\infty]$.

\section{MULTIPLICATIVE PROCESSES AND SMALL-SCALE DYNAMO MODELS}

In previous work [8] we studied the multifractal spectrum of two fast dynamo models. The multiplicative cascade approach allows us both to reproduce and to extend those results.

\section{A. Random cell dynamo}

In the random cell dynamo model [23] a twodimensional lattice was partitioned into cells in which the basic processes of field amplification and reconnection take place in a random fashion. A nonlinear field damping mechanism is used that can be either of prompt or delayed type and diffusion is added via a random walk process controlled by a parameter $0 \leq \eta \leq 0.25$. This model attempts to give a simple description of small-scale magnetic field fluctuations, such as those present in quiet Sun areas.

We calculated the multiplier probability distribution $P(M)$ for the Ohmic dissipation derived from fields generated by the random cell dynamo. The results for both the prompt and delayed damping mechanisms were similar. For the diffusion parameter value $\eta \approx 0.20$ we found good scale invariance, but this was not the case for greater or lesser values of $\eta$. This reproduces the result of our earlier work [8]. The interpretation we gave for this is that, on the one hand, a too small value of $\eta$ represents weak diffusion so that strong field spikes cannot spread into neighboring cells and this inhibits population of the wings of the field probability distribution. On the other hand, a too large value of $\eta$ represents strong diffusion which smooths the field and truncates the wings of the distribution. The value $\eta \approx 0.20$ thus appears to balance these effects and maximizes the intermittence of the generated fields.

The importance of intermittence for this model is further illuminated by the present calculation. For $\eta \approx 0.20$ we find that $P(M)$ is essentially a uniform (flat) distribution plus sharp maxima at $M \approx 0,1$, indicating the presence of a large number of very weak field cells, which appear as holes in the measure. Figure 6(a) gives the $P(M)$ distribution for an image generated by the prompt damping mechanism and with $\eta=0.20$. Figure $6(\mathrm{~b})$ shows the curves that result when noise is artificially added. The noise reduces the number of holes, so the wings of the distribution at $M \approx 0,1$ are suppressed and the curves corresponding to progressively larger scales become progressively more peaked at $M=0.5$. This is the signature behavior of Gaussian noise. Because both are dominated by noise the cellular dynamo plus noise curves in Fig. 6(b) appear to emulate the single-image, quiet Sun case in Fig. 4(b). However, when the noise in the data is removed by averaging the 24 images, we see that the resemblance is
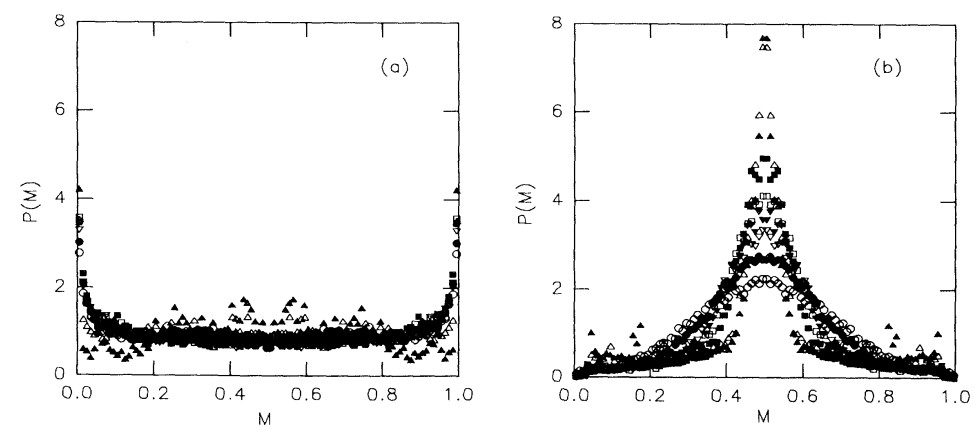

FIG. 6. Cascade multiplier probability distribution density versus multiplier for an image generated by the random cell dynamo with prompt damping mechanism and with diffusivity $\eta=0.20$ for $s=4$ (open circles), 6 (filled circles), 8 (open down triangles), 12 (filled down triangles), 16 (open squares), 24 (filled squares), 32 (open up triangles), and 48 pixels (filled up triangles). (a) Image as generated. (b) Image with added noise. 

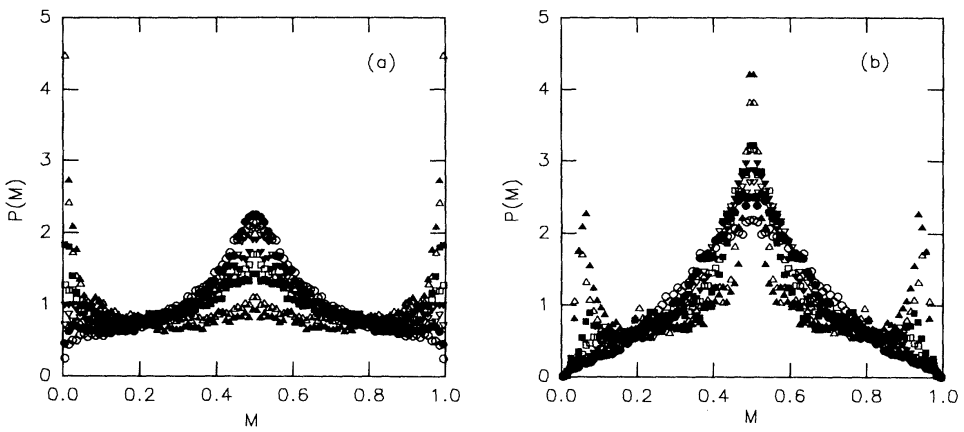

FIG. 7. Cascade multiplier probability distribution density versus multiplier for an image generated by a chaotic ABC flow for $s=4$ (open circles), 6 (filled circles), 8 (open down triangles), 12 (filled down triangles), 16 (open squares), 24 (filled squares), 32 (open up triangles), and 48 pixels (filled up triangles). (a) Image as generated. (b) Image with added noise. only superficial; the real data curve does not resemble at all the curve for the cellular dynamo.

\section{B. Chaotic ABC flow}

We also have studied fields produced by a conducting fluid undergoing a prescribed, externally imposed, but chaotic, ABC-type flow [24]. While it was constructed as a successful demonstration of fast dynamo action, it produces a distribution of very strong fields in a background of weak field fluctuations which can be compared to that of solar active region fields.

Figure 7(a) gives the $P(M)$ distribution for the original image, kindly provided in digital form by D. Galloway. The curves are scale invariant in the intermediate multiplier ranges $(0.1<M<0.4$ and $0.6<M<0.9)$, but the smaller scales have higher peaks at $M \approx 0.5$. Just as in the cellular dynamo case, the presence of a large number of weak field pixels is manifested in the large peaks at $M \approx 0,1$. Figure $7(\mathrm{~b})$, obtained from the generated image with added noise, has lesser peaks at $M \approx 0,1$ and presents the Gaussian behavior of large peaks for large scales in the center of the $P(M)$ distribution. We observe minor peaks at $M \approx 0.05,0.95$ for the large scales. Comparing these curves to those obtained from the solar data, we observe that, even with added noise, the $P(M)$ for the model resembles only slightly that for the data. At the same time, the lack of scale invariance is accentuated.

\section{DISCUSSION AND CONCLUSIONS}

As Fig. 5 illustrates, the approach of random multipliers allows relatively easy calculation of the multifractal spectrum to great accuracy. This includes extension of the curve to negative values of $f(\alpha)$ which represent the effects of very large and very rare fluctuations of the measure. These would be expected to be encountered only in correspondingly large data sets $[10,11]$. This accuracy, however, hinges entirely on how accurately the multiplier probability distribution $P(M)$ actually is known and on how scale invariant it actually is. For real data, this is a matter of degree and of judgment. The $P(M)$ curves in Figs. 3 and 4 illustrate the extent to which this is so in the case of solar data. If the multiplier probability distributions are indeed scale invariant, it has been shown that cascade bases other than the binary one used here will lead to fundamentally the same results [22].
A variety of $P(M)$ distributions can lead to very similar $f(\alpha)$. In principle, this probability distribution can be used to calculate the full multifractal spectrum, but the reverse is not true [25]. Thus the distribution $P(M)$ is the more fundamental quantity. It has the further advantage over the multifractal spectrum of taking only finite values on a finite range. This in turn makes its interpretation in terms of image characteristics more transparent. For example, it is easy to display, if not to interpret, the degree of scale invariance. The probability distribution also is more closely related to the actual physics of the cascade process by which the observed field distributions are presumed to be built up.

As a result of this, the random multiplier approach allows us to tighten further the constraints imposed by the observations on theory. From the point of view of simple scale invariance, the random cell dynamo model with $\eta \approx 0.20$ and the chaotic ABC dynamo seem in agreement with observations. Further, the differences in the $f(\alpha)$ singularity spectra between the models and the data were somewhat subtle. Aside from a degree of scale invariance, however, $P(M)$ from the random cell dynamo model does not resemble that form the data at all. Thus, in our previous work [8] we had concluded that the cellular dynamo multifractal spectrum resembled that of the quiet Sun images. The $P(M)$ distribution, however, provides a more stringent test that the cellular dynamo in its present form does not pass. The same is true for the ABC dynamo. (Its authors were not attempting to model solar field distributions, but rather to demonstrate fast dynamo action.)

A partial understanding of this increased stringency may be offered by the simple fact that the multiplier method works as well as it does for the solar fields. This implies that, although there are spatial correlations at many scales in the field distributions, there are no important correlations among scales in the field formation process. It is our hope that the approach presented here will point the way to improved models and thus lead to an understanding of the generation mechanisms of solar surface magnetism.

We draw attention again to the fact that the multiplier probability distributions from our data resemble that found by Chhabra and Sreenivasan [11] by a similar procedure for one-dimensional data on the dissipation field in a turbulent atmospheric surface layer. Further, the image-to-image fluctuations in the multifractal spectra 
that we have found appear to follow the same, rather restrictive, form of the sample-to-sample fluctuations characteristic of both random multiplier simulations and of atmospheric turbulence [22]. These similarities include images of both quiet Sun and active region fields, made at two observatories by three different techniques, and covering a factor of $\sim 6$ in physical scale. This implies that our results may be generic and therefore of interest not only to solar physicists, but also to a wider scientific community.

\section{ACKNOWLEDGMENTS}

The authors are grateful to Dr. G. A. Chapman and Dr. S. R. Walton for the acquisition and processing of the SFO data. We thank Dr. D. Galloway, Dr. Ch. U. Keller, and Dr. K. P. Topka for making additional images available to us. A.C.C. acknowledges released time provided by the CSUN Research and Grants Committee. This work was supported in part by NSF Grants Nos. ATM-8817634, ATM-9115111, and ATM-9309901.
[1] H. G. E. Hentschel and I. Procaccia, Physica D 8, 435 (1983).

[2] U. Frisch, in Turbulence and Predictability in Geophysical Fluid Dynamics, edited by M. Gil, R. Benzi, and G. Parisi (North-Holland, Amsterdam, 1985).

[3] U. Frisch and G. Parisi, in Turbulence and Predictability in Geophysical Fluid Dynamics (Ref. [2]).

[4] T. C. Halsey, M. H. Jensen, L. P. Kadanoff, I. Procaccia, and B. I. Shraiman, Phys. Rev. A 33, 1141 (1986).

[5] G. Paladin and A. Vulpiani, Phys. Rep. 156, 148 (1987).

[6] G. Meneveau and K. R. Sreenivasan, J. Fluid Mech. 224, 429 (1991).

[7] J. K. Lawrence, A. A. Ruzmaikin, and A. C. Cadavid, Astrophys. J. 417, 850 (1993).

[8] A. C. Cadavid, J. K. Lawrence, A. A. Ruzmaikin, and A. Kayleng-Knight, Astrophys. J. 429, 391 (1994).

[9] B. B. Mandelbrot, The Fractal Geometry of Nature (Freeman, San Francisco, 1982).

[10] A. B. Chhabra and K. R. Sreenivasan, Phys. Rev. A 43, 1114 (1991).

[11] A. B. Chhabra and K. R. Sreenivasan, in New Perspectives in Turbulence, edited by L. Sirovich (Springer, Berlin, 1991).

[12] B. B. Mandelbrot, in Fractals Physical Origin and Properties, edited by L. Pietronero, Ettore Majorana International Science Series Vol. 45 (Plenum, New York, 1989).

[13] G. A. Chapman and S. R. Walton, in High Spatial Resolu- tion Solar Observations, edited by O. van der Luhe (National Solar Observatory, Sunspot, NM, 1989).

[14] J. K. Lawrence, G. A. Chapman, and S. R. Walton, Astrophys. J. 375, 771 (1991).

[15] A. M. Title, T. D. Tarbell, K. P. Topka, S. H. Ferguson, R. A. Shine, and the SOUP Team, Astrophys. J. 336, 475 (1989).

[16] C. U. Keller, Nature 359, 307 (1992).

[17] E. Ott, Y. Du, K. R. Sreenivasan, A. Juneja, and A. K. Suri, Phys. Rev. Lett. 69, 2654 (1992).

[18] C. J. G. Evertsz and B. B. Mandelbrot, in Chaos and Fractals: New Frontiers in Science, edited by H.-O. Peitgen, H. Jürgens, and D. Saupe (Springer, Berlin, 1992).

[19] C. Meneveau and K. R. Sreenivasan, Phys. Lett. A 137, 103 (1989).

[20] H. C. Spruit, in The Sun as a Star, edited by S. Jordan (NASA, Washington, D.C., 1981), NASA Monograph Series SP-450.

[21] B. B. Mandelbrot, J. Fluid Mech. 62, 331 (1974).

[22] A. B. Chhabra and K. R. Sreenivasan, Phys. Rev. Lett. 68, 2762 (1992).

[23] A. A. Ruzmaikin, P. C. Liewer, and J. Feynman, Geophys. Astrophys. Fluid Dynam. 73, 163 (1993).

[24] D. J. Galloway and M. R. E. Proctor, Nature 356, 691 (1992).

[25] A. B. Chhabra, C. Jensen, and K. R. Sreenivasan, Phys. Rev. A 40, 4953 (1989). 

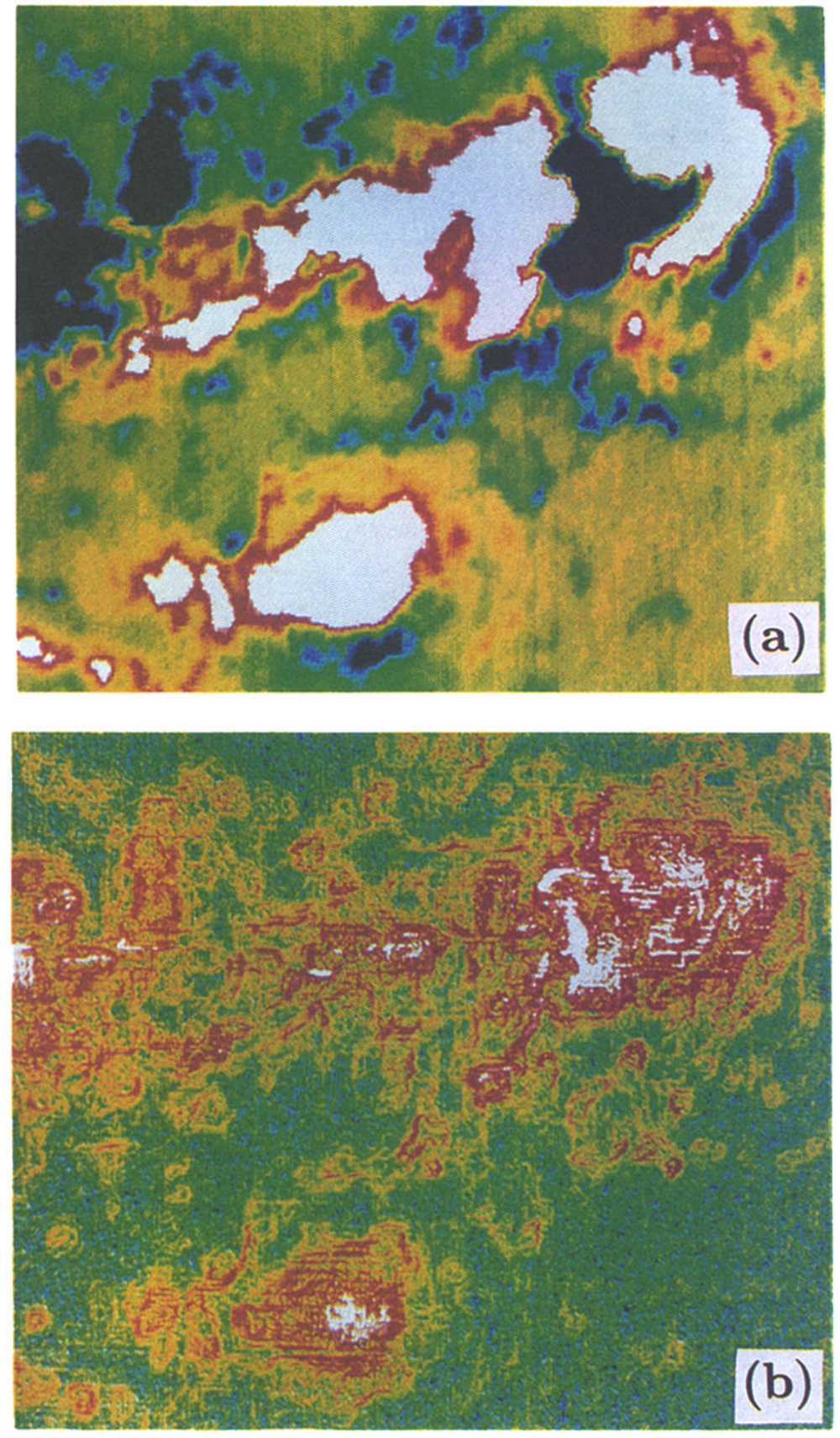

FIG. 1. (a) False color, line-of-sight magnetic field image of NOAA active regions 6850 and 6853 made with the San Fernando Observatory video spectra-spectroheliograph system on 3 October 1991 at UT 20:03:30. The spatial sale is 0.46 arcsec pixel $^{-1}$, corresponding to about $340 \mathrm{~km}$ on the sun. Spatial resolution is seeing limited at $\geq 1$ arcsec. The field of view is $\sim 200$ arcsec or $\sim 150000 \mathrm{~km}$ on the sun. White (black) represents positive (negative) magnetic polarity. North is up, east is left. (b) Ohmic dissipation measure derived from image (a) via Eq. (3.7). 